\title{
Nucleon and Nucleon-to- $\Delta$ Axial Form Factors from Lattice QCD
}

\section{Constantia Alexandrou}

Department of Physics, University of Cyprus, CY-1678 Nicosia, Cyprus

E-mail: alexandeucy.ac.cy

\section{Giannis Koutsou}

Department of Physics, University of Cyprus, CY-1678 Nicosia, Cyprus

E-mail: koutsoueucy.ac.cy

\section{Theodoros Leontiou}

Department of Physics, University of Cyprus, CY-1678 Nicosia, Cyprus

E-mail: t.leontioulucy.ac.cy

\section{John W. Negele}

Center for Theoretical Physics, Laboratory for Nuclear Science and Department of Physics,

Massachusetts Institute of Technology, Cambridge, Massachusetts 02139, U.S.A.

E-mail: negele@mit.edu

\section{Antonios Tsapalis*}

University of Athens, Institute of Accelerating Systems and Applications, Athens, Greece

E-mail: a.tsapaliseiasa.gr

We present results on the nucleon axial vector form factors $G_{A}\left(q^{2}\right)$ and $G_{p}\left(q^{2}\right)$ in the quenched theory and using two degenerate flavors of dynamical Wilson fermions for momentum transfer squared from about 0.1 to about $2 \mathrm{GeV}^{2}$ and for pion masses in the range of 380 to $600 \mathrm{MeV}$. We also present results on the corresponding $N$ to $\Delta$ axial vector transition form factors $C_{5}^{A}\left(q^{2}\right)$ and $C_{6}^{A}\left(q^{2}\right)$ using, in addition to Wilson fermions, domain wall valence quarks and dynamical staggered sea quarks provided by the MILC collaboration.

The XXV International Symposium on Lattice Field Theory

July 30 - August 42007

Regensburg, Germany

\footnotetext{
* Speaker.
} 


\section{Introduction}

Form factors are fundamental quantities which characterize important features of the hadrons such as their size, charge distribution and magnetization. In this work we present a calculation of the axial form factors of the nucleon and the nucleon $(\mathrm{N})$ to $\Delta$ transition [1]. Spontaneous breaking of axial symmetry in QCD is manifest in the existence of light pseudo-Goldstone bosons. The smallness of the pion mass is connected to the axial symmetry breaking through the partial conservation of axial current (PCAC) hypothesis, which relates the divergence of the axial vector current to the pion field. This translates into the pion-pole dominance of the induced nucleon pseudoscalar form factor, $G_{p}\left(q^{2}\right)$. Furthermore, it relates the nucleon axial charge $g_{A}$, to the $\pi N N$ coupling, $g_{\pi N N}$ via the well known Goldberger-Treiman relation (GTR), $m_{N} g_{A}=f_{\pi} g_{\pi N N}$, where $f_{\pi}$ is the pion decay constant and $m_{N}$ the mass of the nucleon. Similarly, PCAC applied to the $\mathrm{N}$ to $\Delta$ transition, leads to the assumption of pion-pole dominance in the case of $C_{6}^{A}$ and to the non-diagonal GTR, $2 m_{N} C_{5}^{A}=f_{\pi} g_{\pi N \Delta}$, where $g_{\pi N \Delta}$ is the strong coupling constant associated with the $\pi N \Delta$ vertex. Our calculation of the nucleon axial vector form factors, $G_{A}\left(q^{2}\right)$ and $G_{p}\left(q^{2}\right)$, as well as the pseudoscalar form factor $G_{\pi N N}$, for a range of pion masses down to $380 \mathrm{MeV}$, both in the quenched theory and using two degenerate flavors of dynamical Wilson fermions, enables us to check pion-pole dominance and the Goldberger-Treiman relation. Similarly, in the case of the $\mathrm{N}$ to $\Delta(1232)$ weak current transition, we evaluate the corresponding dominant form factors $C_{5}^{A}\left(q^{2}\right)$ and $C_{6}^{A}\left(q^{2}\right)$, as well as the pseudoscalar form factor $G_{\pi N \Delta}$, using besides Wilson fermions, dynamical staggered quark configurations generated by the MILC collaboration and domain wall fermions for pion masses as low as $360 \mathrm{MeV}$. The evaluation of both nucleon and $\mathrm{N}$ to $\Delta$ transition form factors enables us to check the $q^{2}$-dependence of ratios of transition to nucleon form factors, such as $G_{\pi N \Delta}\left(q^{2}\right) / G_{\pi N N}\left(q^{2}\right)$ and $C_{5}^{A}\left(q^{2}\right) / G_{A}\left(q^{2}\right)$, which show a weaker quark mass dependence and are more likely to show less sensitivity to lattice systematics.

\section{Axial Form Factors and the Pion Pole}

We take the $\mathrm{u}$ - and the d- quarks to be degenerate and work in terms of the fermion isospin doublet $\psi$. The axial vector and pseudoscalar currents are given by $A_{\mu}^{a}(x)=\bar{\psi}(x) \gamma_{\mu} \gamma_{5} \frac{\tau^{a}}{2} \psi(x)$ and $P^{a}(x)=\bar{\psi}(x) \gamma_{5} \frac{\tau^{a}}{2} \psi(x)$ respectively, where $\tau^{a}$ are Pauli-matrices acting in flavor space. The matrix element of the weak axial vector current between nucleon states is written as

$$
\left\langle N\left(p^{\prime}, s^{\prime}\right)\left|A_{\mu}^{3}\right| N(p, s)\right\rangle=i\left(\frac{m_{N}^{2}}{E_{N}\left(p^{\prime}\right) E_{N}(p)}\right)^{1 / 2} \bar{u}\left(p^{\prime}, s^{\prime}\right)\left[\left(G_{A}\left(q^{2}\right) \gamma_{\mu} \gamma_{5}+\frac{q_{\mu}}{2 m_{N}} G_{p}\left(q^{2}\right)\right)\right] \frac{\tau^{3}}{2} u(p, s)
$$

with the axial vector, $G_{A}\left(q^{2}\right)$, and the induced pseudoscalar form factor, $G_{p}\left(q^{2}\right)$, being functions of the invariant momentum transfer squared, $q^{2}=\left(p^{\prime}-p\right)^{2}$. Experimentally, the axial charge of the nucleon, $g_{A} \equiv G_{A}(0)$ is very well known from the neutron $\beta$-decay and takes the value $g_{A}=1.2695(29)$. The momentum dependence of $G_{A}$ has been extracted from pion electroproduction or quasielastic neutrino scattering experiments (see Ref. [2] for reviews) and it is conventionally parameterized by a dipole form, $G_{A}\left(q^{2}\right)=g_{A} /\left(1-\frac{q^{2}}{M_{A}^{2}}\right)^{2}$, with an axial mass, $M_{A}=$ $1.026 \pm 0.0021 \mathrm{GeV}$. The induced pseudoscalar form factor $G_{p}\left(q^{2}\right)$ is less well studied experimentally [2], with ordinary and radiative muon capture experiments giving different results. Both form

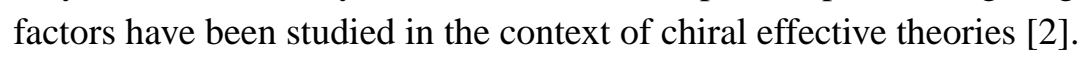


In QCD the explicit breaking of axial current conservation leads to the axial Ward-Takahashi identity, $\partial^{\mu} A_{\mu}^{a}=2 m_{q} P^{a}$, where $m_{q}$ is the renormalized quark mass. Using PCAC we have the relation, $\partial^{\mu} A_{\mu}^{a}=f_{\pi} m_{\pi}^{2} \pi^{a}$, and therefore the pion field is expressed in terms of the pseudoscalar current as $\pi^{a}=2 m_{q} P^{a} / f_{\pi} m_{\pi}^{2}$. Taking the matrix element of the pseudoscalar density between nucleon states, the $\pi N N$ form factor is obtained via

$$
2 m_{q}<N\left(p^{\prime}, s^{\prime}\right)\left|P^{3}\right| N(p, s)>=\left(\frac{m_{N}^{2}}{E_{N}\left(\mathbf{p}^{\prime}\right) E_{N}(\mathbf{p})}\right)^{1 / 2} \frac{f_{\pi} m_{\pi}^{2} G_{\pi N N}\left(q^{2}\right)}{m_{\pi}^{2}-q^{2}} \bar{u}\left(p^{\prime}, s^{\prime}\right) i \gamma_{5} \frac{\tau^{3}}{2} u(p, s) .
$$

Using the PCAC hypothesis together with Eq. (2.2) we obtain the diagonal Goldberger-Treiman relation

$$
G_{A}\left(q^{2}\right)+\frac{q^{2}}{4 m_{N}^{2}} G_{p}\left(q^{2}\right)=\frac{1}{2 m_{N}} \frac{2 G_{\pi N N}\left(q^{2}\right) f_{\pi} m_{\pi}^{2}}{m_{\pi}^{2}-q^{2}}
$$

In the chiral limit, the pole on the right hand size of Eq. (2.3) must be compensated by a similar singularity in $G_{p}\left(q^{2}\right)$ since $G_{A}(0)$ is finite. Therefore, assuming pion-pole dominance, $G_{p}\left(q^{2}\right)$ is given by

$$
\frac{1}{2 m_{N}} G_{p}\left(q^{2}\right) \sim \frac{2 G_{\pi N N}\left(q^{2}\right) f_{\pi}}{m_{\pi}^{2}-q^{2}}
$$

and substituting in Eq. (2.3) we obtain the well known relation, $m_{N} G_{A}\left(q^{2}\right)=f_{\pi} G_{\pi N N}\left(q^{2}\right)$.

The invariant proton to $\Delta^{+}$weak matrix element is expressed in terms of four transition form factors as

$$
\begin{gathered}
<\Delta\left(p^{\prime}, s^{\prime}\right)\left|A_{\mu}^{3}\right| N(p, s)>=i \sqrt{\frac{2}{3}}\left(\frac{m_{\Delta} m_{N}}{E_{\Delta}\left(\mathbf{p}^{\prime}\right) E_{N}(\mathbf{p})}\right)^{1 / 2} \bar{u}_{\Delta^{+}}^{\lambda}\left(p^{\prime}, s^{\prime}\right) \\
{\left[\left(\frac{C_{3}^{A}\left(q^{2}\right)}{m_{N}} \gamma^{\nu}+\frac{C_{4}^{A}\left(q^{2}\right)}{m_{N}^{2}} p^{\prime \nu}\right)\left(g_{\lambda \mu} g_{\rho \nu}-g_{\lambda \rho} g_{\mu \nu}\right) q^{\rho}+C_{5}^{A}\left(q^{2}\right) g_{\lambda \mu}+\frac{C_{6}^{A}\left(q^{2}\right)}{m_{N}^{2}} q_{\lambda} q_{\mu}\right] u_{P}(p, s) .}
\end{gathered}
$$

The form factors $C_{3}^{A}\left(q^{2}\right)$ and $C_{4}^{A}\left(q^{2}\right)$ belong to the transverse part of the axial current and, as was recently demonstrated [B], both are suppressed relative to $C_{5}^{A}\left(q^{2}\right)$ and $C_{6}^{A}\left(q^{2}\right)$. The pseudoscalar transition form factor, $G_{\pi N \Delta}$, is defined similarly to $G_{\pi N N}$, via

$$
2 m_{q}<\Delta\left(p^{\prime}, s^{\prime}\right)\left|P^{3}\right| N(p, s)>=i \sqrt{\frac{2}{3}}\left(\frac{m_{\Delta} m_{N}}{E_{\Delta}\left(\mathbf{p}^{\prime}\right) E_{N}(\mathbf{p})}\right)^{1 / 2} \frac{f_{\pi} m_{\pi}^{2} G_{\pi N \Delta}\left(q^{2}\right)}{m_{\pi}^{2}-q^{2}} \bar{u}_{\Delta^{+}}^{v}\left(p^{\prime}, s^{\prime}\right) \frac{q_{v}}{2 m_{N}} u_{P}(p, s)
$$

The $\pi N N$ and $\pi N \Delta$ coupling constants are defined at $q^{2}=m_{\pi}^{2}$ as $g_{\pi N N}=G_{\pi N N}\left(m_{\pi}^{2}\right)$ and $g_{\pi N \Delta}=$ $G_{\pi N \Delta}\left(m_{\pi}^{2}\right)$. The dominant form factors $C_{5}^{A}\left(q^{2}\right)$ and $C_{6}^{A}\left(q^{2}\right)$, which belong to the longitudinal part of the axial current, are related via PCAC to $G_{\pi N \Delta}\left(q^{2}\right)$ :

$$
C_{5}^{A}\left(q^{2}\right)+\frac{q^{2}}{m_{N}^{2}} C_{6}^{A}\left(q^{2}\right)=\frac{1}{2 m_{N}} \frac{G_{\pi N \Delta}\left(q^{2}\right) f_{\pi} m_{\pi}^{2}}{m_{\pi}^{2}-q^{2}},
$$

which is known as the non-diagonal GTR. Pion-pole dominance for $C_{6}^{A}\left(q^{2}\right)$, as for $G_{p}\left(q^{2}\right)$, leads to the relations $C_{6}^{A}\left(q^{2}\right) \sim m_{N} G_{\pi N \Delta}\left(q^{2}\right) f_{\pi} / 2\left(m_{\pi}^{2}-q^{2}\right)$ and $2 m_{N} C_{5}^{A}\left(q^{2}\right)=f_{\pi} G_{\pi N \Delta}\left(q^{2}\right)$. It is clear from the above relations that $C_{5}^{A}$ is analogous to $G_{A}$ and $C_{6}^{A}$ analogous to $G_{p}$. 


\section{Lattice techniques}

The techniques used for the evaluation of the axial form factors were developed for the study of the electromagnetic form factors [ 7 , 5, 6]. In the evaluation of the three-point functions involved in the calculation of the electromagnetic nucleon and $\mathrm{N}$ to $\Delta$ transition form factors we use sequential inversion through the sink, thereby obtaining the form factors for all momentum transfers. This method requires fixing the source-sink time separation, as well as the initial and final hadron states, but allows the insertion of any operator with arbitrary momentum at any time slice. An important ingredient of our method is the construction of optimal sources and sinks using a linear combination of interpolating fields. It was shown in Refs. [1, 3] that, for axial operators, the most symmetric linear combination of matrix elements that can be considered is

$$
\sum_{k=1}^{3} \Pi^{A}\left(\mathbf{0},-\mathbf{q} ; \Gamma_{k} ; \mu=j\right)=i \frac{C}{4 m_{N}}\left[\left(E_{N}+m_{N}\right)\left(\delta_{1, j}+\delta_{2, j}+\delta_{3, j}\right) G_{A}\left(Q^{2}\right)-\left(q_{1}+q_{2}+q_{3}\right) \frac{q_{j}}{2 m_{N}} G_{p}\left(Q^{2}\right)\right],
$$

where $j=1,2,3$ labels the spatial current direction, $\Gamma$ projects to definite nucleon spin states and $C$ is a kinematical factor. $\Pi^{A}\left(\mathbf{p}^{\prime}, \mathbf{p} ; \Gamma ; \mu\right)$ is the large Euclidean time limit of an appropriately constructed ratio of the three-point function to two-point functions, in which time dependencies of the time evolution and overlaps of the initial trial state and the nucleon state cancel. Since this optimized sink, involving momentum in all spatial directions, coincides with the one calculated for the electromagnetic current no new sequential inversions are required for the axial vector current. The same holds for the pseudoscalar current and the $\mathrm{N}$ to $\Delta$ optimized sinks. As indicated in Eq. (3.1), we use kinematics where the final nucleon is produced at rest and therefore the momentum transfer $\mathbf{q}=\mathbf{p}^{\prime}-\mathbf{p}=-\mathbf{p}$. We take $-q^{2}=Q^{2}>0$ with $Q^{2}$ being the Euclidean momentum transfer squared.

\begin{tabular}{|c|c|c|c|c|}
\hline \# confs & $\kappa$ or $a m_{l}$ & $m_{\pi}(\mathrm{GeV})$ & $M_{N}(\mathrm{GeV})$ & $M_{\Delta}(\mathrm{GeV})$ \\
\hline \multicolumn{5}{|c|}{ Quenched $32^{3} \times 64 \quad a^{-1}=2.14(6) \mathrm{GeV}$} \\
\hline 200 & 0.1554 & $0.563(4)$ & $1.267(11)$ & $1.470(15)$ \\
\hline 200 & 0.15 & $0.490(4)$ & $1.190(13)$ & $1.425(16)$ \\
\hline \multirow[t]{2}{*}{200} & 0.1 & $0.411(4)$ & $1.109(13)$ & 1.38 \\
\hline & $\kappa_{c}=0.1571$ & 0 . & $0.938(9)$ & \\
\hline \multicolumn{3}{|c|}{ Unquenched Wilson $24^{3} \times 40$ [7] } & \multicolumn{2}{|c|}{$a^{-1}=2.56(10) \mathrm{GeV}$} \\
\hline 185 & 0.15 & $0.691(8)$ & $1.485(18)$ & $1.687(15)$ \\
\hline 157 & 0.1580 & $0.509(8)$ & $1.280(26)$ & $1.559(19)$ \\
\hline \multicolumn{3}{|c|}{ Unquenched Wilson $24^{3} \times 32$ [8] } & \multicolumn{2}{|c|}{$a^{-1}=2.56(10) \mathrm{GeV}$} \\
\hline \multirow[t]{2}{*}{200} & 0.15825 & $0.384(8)$ & $1.083(18)$ & $1.395(18)$ \\
\hline & $\kappa_{c}=0.1585$ & 0 . & $0.938(33)$ & \\
\hline \multicolumn{5}{|c|}{ MILC $20^{3} \times 64 \quad a^{-1}=1.58 \mathrm{GeV}$} \\
\hline 200 & & $0.594(1)$ & $1.416(20)$ & 1.68 \\
\hline 198 & 0.02 & $0.498(3)$ & 1.261 & $1.589(35)$ \\
\hline 100 & 0.01 & $0.362(5)$ & $1.139(25)$ & $1.488(71)$ \\
\hline \multicolumn{5}{|c|}{ MILC $28^{3} \times 64 \quad a^{-1}=1.58 \mathrm{GeV}$} \\
\hline 150 & 0.01 & $0.357(2)$ & $1.210(24)$ & $1.514(41)$ \\
\hline
\end{tabular}

Table 1: The number of configurations, the hopping parameter, $\kappa$, for the case of Wilson fermions or the mass of the light quarks, $m_{l}$, for the MILC staggered quarks, and the pion, nucleon and $\Delta$ masses. 
In Table I we collect the parameters for our calculation. In the so called hybrid approach, that uses staggered sea quarks and domain wall valence quarks, we have no $\mathscr{O}(a)$ artifacts, unlike Wilson fermions where cutoff effects are $\mathscr{O}(a)$. We use the same parameters for the domain wall operator as those used in Ref. [9], namely we take the length of the fifth dimension, $L_{5} / a=16$ and the valence quark mass that is tuned to reproduce the pion mass calculated with the staggered quark action. Finite volume effects can be assessed by comparing results at the lowest pion mass. It was shown [1] that results on the $20^{3}$ spatial volume were consistent with the results on the $28^{3}$ lattice. The source-sink separation is optimized so that, on the one hand, it is sufficiently large to ensure ground state dominance and, on the other, small enough so that gauge noise is kept at a minimum. In all cases, we use the non-perturbatively determined value for the axial renormalization constant, $Z_{A}$. Note that the pseudoscalar renormalization constant is not needed for the quantities under consideration here.

\section{Results}

We first consider the ratios $G_{\pi N \Delta}\left(Q^{2}\right) / G_{\pi N N}\left(Q^{2}\right)$ and $2 C_{5}^{A}\left(Q^{2}\right) / G_{A}\left(Q^{2}\right)$. We note that, in the ratio $G_{\pi N \Delta}\left(Q^{2}\right) / G_{\pi N N}\left(Q^{2}\right)$, the renormalized quark mass cancels eliminating one source of systematic error.

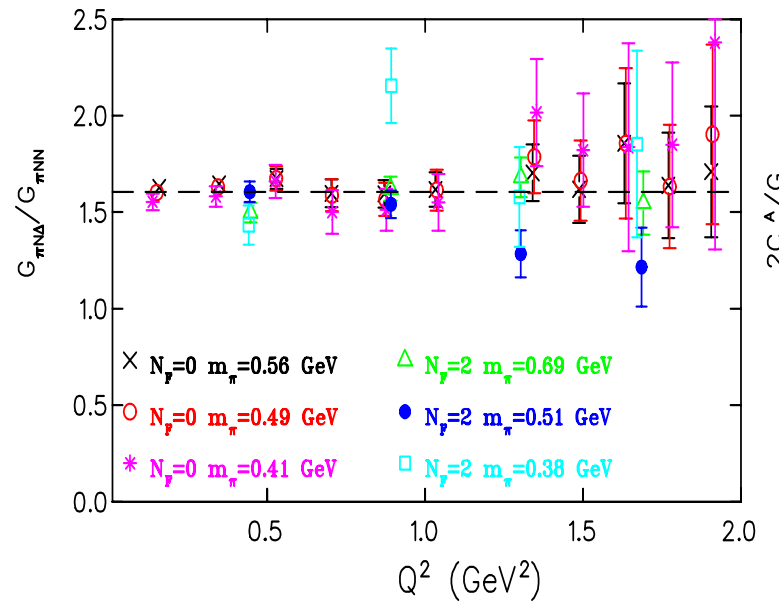

Figure 1: The ratio of form factors $G_{\pi N \Delta}\left(Q^{2}\right) / G_{\pi N N}\left(Q^{2}\right)$ as a function of $Q^{2}$ for Wilson fermions for the quenched theory, denoted by $N_{F}=0$, and for two dynamical Wilson quarks, denoted by $N_{F}=2$.

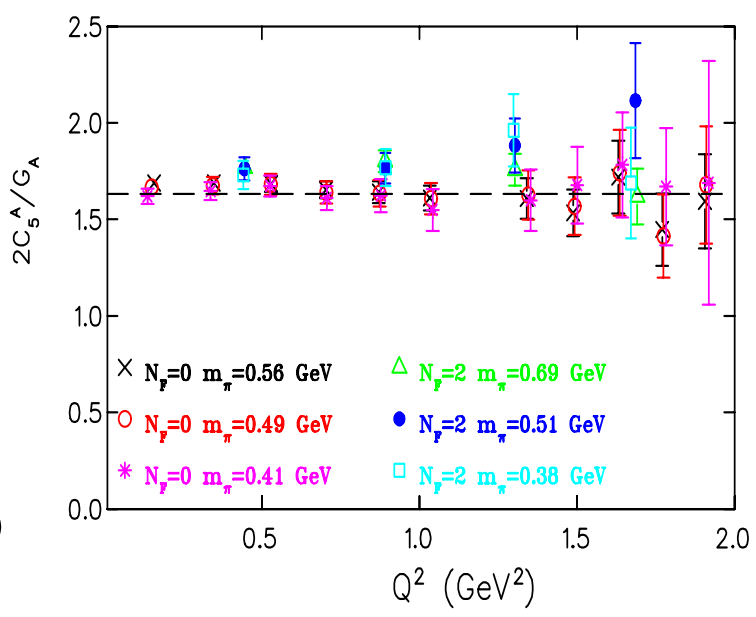

Figure 2: The ratio of $2 C_{5}^{A}\left(Q^{2}\right) / G_{A}\left(Q^{2}\right)$ as a function of $Q^{2}$. The notation is the same as that of Fig. 1.

As can be seen in Figs. 1 and 1 , these ratios show weak dependence on the quark mass and are therefore more suited for comparison with physical results. Both these ratios are $Q^{2}$ independent and can be thus fitted to a constant. Fitting the quenched data, which carry the smallest statistical errors, we obtain the value of 1.60(2) shown by the dashed line. Taking the ratio of the diagonal and non-diagonal GTRs $m_{N} G_{A}=f_{\pi} G_{\pi N N}$ and $2 m_{N} C_{5}^{A}=f_{\pi} G_{\pi N \Delta}$, we find that $G_{\pi N \Delta}\left(Q^{2}\right) / G_{\pi N N}\left(Q^{2}\right)=2 C_{5}^{A}\left(Q^{2}\right) / G_{A}\left(Q^{2}\right)$. In Fig. 目, we show the ratio $2 C_{5}^{A}\left(Q^{2}\right) / G_{A}\left(Q^{2}\right)$, which is indeed also $Q^{2}$ independent, and fitting to the quenched data we find the value of 1.63(1) shown 
by the dashed line. They are also approximately equal in the unquenched case [1]. Therefore, on the level of ratios, the GTRs are satisfied.

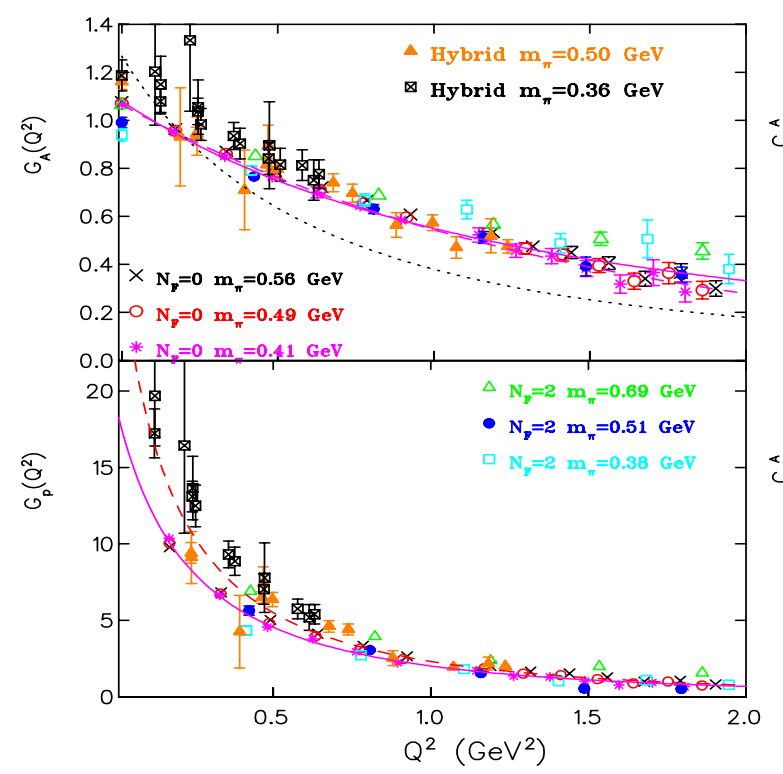

Figure 3: $G_{A}\left(Q^{2}\right)$ (upper) and $G_{p}\left(Q^{2}\right)$ (lower) for the quenched theory and for two dynamical Wilson fermions. Results in the hybrid approach are from [9].

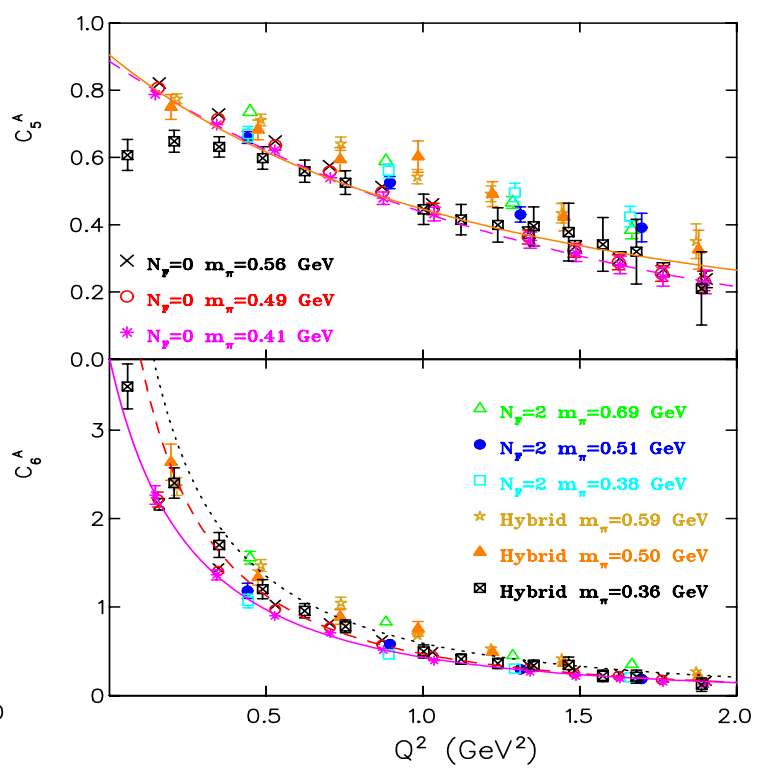

Figure 4: $C_{5}^{A}\left(Q^{2}\right)$ (upper) and $C_{6}^{A}\left(Q^{2}\right)$ (lower) for Wilson fermions and in the hybrid approach.

In Fig. 3, we show our results for the nucleon axial form factors using Wilson fermions. We also include recent results obtained in the hybrid approach [9] using similar parameters to those used in our evaluation of the $\mathrm{N}$ to $\Delta$ axial form factors. The main observation is that, at the smallest domain wall quark mass, results in the hybrid approach deviate from quenched results. In particular, we note that the value of the nucleon axial charge $g_{A}$ becomes larger in the hybrid scheme approaching the experimental value. The dotted line shows the dipole fit to the experimental data. As can be seen, lattice results fall off slower than experiment. However, the large deviations observed at low $Q^{2}$ for a pion mass of about $360 \mathrm{MeV}$ point to large pion cloud effects, which tend to increase the form factors at small $Q^{2}$. The solid line is a dipole fit of the quenched $G_{A}\left(Q^{2}\right)$ at a pion mass of $411 \mathrm{MeV}$, yielding an axial mass $M_{A}=1.58(3) \mathrm{GeV}$. We note that a fit to an exponential of the form $\tilde{g}_{0} \exp \left(-Q^{2} / \tilde{m}_{A}^{2}\right)$, also describes the lattice data, yielding a curve that is indistinguishable from the dipole fit.

Very similar behavior is observed for the transition form factor $C_{5}^{A}\left(Q^{2}\right)$ shown in Fig. $\bigoplus$, where a dipole fit, shown with the solid line, provides a good description of the lattice results. Again the axial mass obtained by fitting the quenched results at the lowest mass is larger than the value of $1.28(10) \mathrm{GeV}$ extracted from available experimental results [10]. A fit to an exponential form also provides a good fit to the lattice data.

Having fitted $G_{A}\left(Q^{2}\right)$ and $C_{5}^{A}\left(Q^{2}\right)$, the $Q^{2}$-dependence for the form factors $G_{p}\left(Q^{2}\right)$ and $C_{6}^{A}\left(Q^{2}\right)$ can be obtained using pion-pole dominance. The resulting curves are shown by the dashed lines in Figs. 3 and $\$$ for the quenched theory at the lowest pion mass and show deviations at low $Q^{2}$. The dotted line is the corresponding result in the hybrid approach at the smallest pion mass. In addition 
we show curves that are obtained using

$$
\frac{G_{p}\left(Q^{2}\right)}{G_{A}\left(Q^{2}\right)}=\frac{g_{0}}{\left(Q^{2} / m^{2}+1\right)}
$$

with a corresponding expression for $C_{6}^{A}\left(Q^{2}\right) / C_{5}^{A}\left(Q^{2}\right)$, where $g_{0}$ and $m$ are treated as fit parameters. As expected, this provides a good description of the $Q^{2}$-dependence for $G_{p}\left(Q^{2}\right)$ and $C_{6}^{A}\left(Q^{2}\right)$ as shown by the solid lines, which correspond to the fits of the quenched data at the lowest pion mass.

\section{Conclusions}

We present results for the nucleon axial vector form factors $G_{A}\left(Q^{2}\right)$ and $G_{p}\left(Q^{2}\right)$, as well as for the corresponding $\mathrm{N}$ to $\Delta$ axial transition form factors $C_{5}^{A}\left(Q^{2}\right)$ and $C_{6}^{A}\left(Q^{2}\right)$. The $\pi N N$ and $\pi N \Delta$ form factors $G_{\pi N N}\left(Q^{2}\right)$ and $G_{\pi N \Delta}\left(Q^{2}\right)$ are also evaluated. One of the main conclusions is that $G_{\pi N N}$ and $G_{\pi N \Delta}$ have the same $Q^{2}$ dependence yielding a ratio of $G_{\pi N \Delta}\left(Q^{2}\right) / G_{\pi N N}\left(Q^{2}\right)=1.60(2)$ in good agreement with what is expected phenomenologically. The ratio $2 C_{5}^{A}\left(Q^{2}\right) / G_{A}\left(Q^{2}\right)=1.63(1)$ is also independent of $Q^{2}$. Equality of these two ratios implies the Goldberger-Treiman relations. We also studied the $Q^{2}$-dependence of the form factors separately. Comparing quenched and unquenched results at pion mass of about $360 \mathrm{MeV}$, we observe large unquenching effects on the low $Q^{2}$-dependence of the four form factors, $G_{A}\left(Q^{2}\right), G_{P}\left(Q^{2}\right), C_{5}^{A}\left(Q^{2}\right)$ and $C_{6}^{A}\left(Q^{2}\right)$. This confirms the expectation that pion cloud effects are expected to be large at low $Q^{2}$. Further study of pion cloud effects using lighter quark masses on a finer lattice is called for.

Acknowledgments: A.T. acknowledges support by the University of Cyprus and the program "Pythagoras" of the Greek Ministry of Education. This work is supported in part by the EU Integrated Infrastructure Initiative Hadron Physics (I3HP) under contract RII3-CT-2004-506078 and by the U.S. Department of Energy Office of Nuclear Physics under contract DE-FG02-94ER40818. Computer resources were provided by NERSC under contract No. DE-AC03-76SF00098.

\section{References}

[1] C. Alexandrou et al., Phys. Rev. D (in press), arXiv:0705.4295.

[2] V. Bernard, L. Elouadrhiri and U.-G. Meissner, J. Phys. G 28, R1 (2002) ; T. Gorringe and H. W. Fearing, Rev. Mod. Phys. 76, 31 (2004).

[3] C. Alexandrou, Th. Leontiou, J. W. Negele and A. Tsapalis, Phys. Rev. Lett. 98, 052003 (2006); C. Alexandrou et al., PoS LAT2006, 115 (2006).

[4] C. Alexandrou, G. Koutsou, J. W. Negele and A. Tsapalis, Phys. Rev. D 74, 034508 (2006).

[5] C. Alexandrou et al., Phys. Rev. Lett. 94, 021601 (2005).

[6] C. Alexandrou et al., Nucl. Phys. Proc. Suppl. 129, 302 (2004); C. Alexandrou, Nucl. Phys. Proc. Suppl. 128, 1 (2004); C. Alexandrou et al., Nucl. Phys. Proc. Suppl. 140, 293 (2005); C. Alexandrou et al., PoS LAT2005, 091 (2006).

[7] B. Orth, Th. Lippert and K. Schilling (T $\chi$ L Collaboration), Phys. Rev. D 72, 014503 (2005).

[8] C.Urbach et al., Comput. Phys. Commun. 174, 87(2006).

[9] Ph. Hägler et al., arXiv:0705.4295 ; R. G. Edwards et al., PoS LAT2006, 121 (2006).

[10] T. Kitagaki et al., Phys. Rev. D 42, 1331 (1990). 